\title{
Towards Loop-Free Forwarding of Anonymous Internet Datagrams that Enforce Provenance
}

\author{
J.J. Garcia-Luna-Aceves ${ }^{1,2}$ \\ ${ }^{1}$ Department of Computer Engineering, University of California, Santa Cruz, CA 95064 \\ ${ }^{2}$ Palo Alto Research Center, Palo Alto, CA 94304 \\ Email: jj@soe.ucsc.edu
}

\begin{abstract}
The way in which addressing and forwarding are implemented in the Internet constitutes one of its biggest privacy and security challenges. The fact that source addresses in Internet datagrams cannot be trusted makes the IP Internet inherently vulnerable to DoS and DDoS attacks. The Internet forwarding plane is open to attacks to the privacy of datagram sources, because source addresses in Internet datagrams have global scope. The fact an Internet datagrams are forwarded based solely on the destination addresses stated in datagram headers and the next hops stored in the forwarding information bases (FIB) of relaying routers allows Internet datagrams to traverse loops, which wastes resources and leaves the Internet open to further attacks. We introduce PEAR (Provenance Enforcement through Addressing and Routing), a new approach for addressing and forwarding of Internet datagrams that enables anonymous forwarding of Internet datagrams, eliminates many of the existing DDoS attacks on the IP Internet, and prevents Internet datagrams from looping, even in the presence of routing-table loops.
\end{abstract}

\section{INTRODUCTION}

One of the biggest challenges facing the future of the Internet is that its vulnerabilities to DoS and DDoS attacks are inherent in the algorithms used in the Internet to: (a) assign addresses to hosts, routers, and devices; (b) include source addresses in Internet datagrams; (c) map addresses to routes; (d) bind names to locations in the Internet; and (e) forward Internet datagrams.

In theory, the goal of assigning Internet Protocol (IP) addresses to entities and including the source and destination IP address in each datagram is to have system-friendly identifiers that: state the origin and destination of Internet datagrams based on topological locations where content, services, or devices are made available; and can be matched efficiently against stored information by routers and end systems. However, an IP address simply denotes the point of attachment of a host or router to a network with a given IP address range, without any topological information other than the aggregation of IP addresses. Furthermore, IP addresses are assigned to entities independently of the establishment of routes to services, content, devices, groups, or any entity in general. As a result, routing protocols (e.g., OSPF, BGP) and directory services (e.g., DNS) map names used to denote entities (e.g., domain names) to names that denote points of attachment to networks (IP addresses). In addition to this, the Internet surrenders any control of the allocation of source IP addresses to Internet datagrams, because the origin of an
Internet datagram specifies the source address of the datagram independently of any forwarding mechanism and end nodes are allowed to specify IP source addresses.

Because of the algorithms used to assign IP addresses to entities and write source addresses into Internet datagrams, the source address of an Internet datagram fails to convey its provenance correctly. The recipient of an Internet datagram is unable to authenticate the claimed IP address of the source of the datagram based solely on the basic operation of the forwarding plane of the IP Internet. The receivers of Internet datagrams are forced to use additional mechanisms and information to cope with the fact that a source address need not denote the valid provenance of an Internet datagram. Furthermore, these mechanisms are far more complex than the simple mechanism used by sources of Internet datagrams to state the origins of datagrams. In addition, IP addresses are globally unique and assigned on a long-term basis, which makes it easier for attackers to plan and mount attacks. This constitutes a major vulnerability to DDoS attacks in the current Internet architecture.

In addition to the above, a router forwards an Internet datagram to its next hop based solely on the destination address stated in the datagram and the next hop listed in its forwarding information base (FIB). This is a problem in the presence of routing-table loops, because it is possible for Internet datagrams to traverse loops. The only approach used today is to include a time-to-live (TTL) field in the datagram header that is decremented at each hop of the path traversed by the datagram, and to drop an Internet datagram after the TTL value reaches zero.

The contribution of this paper is to present a set of algorithms that we call PEAR (Provenance Enforcement through Addressing and Routing), and which prevent Internet datagrams from traversing forwarding loops, makes the identity of the origin of an Internet datagram anonymous to the rest of the Internet, and enforces the provenance of an Internet datagram.

Section [I] summarizes current defenses against DDoS flooding attacks. The main objective of this review of prior work is to point out that defending against large DDoS flooding attacks is virtually impossible without changing the basic algorithms used for the allocation of addresses to Internet datagrams, the mapping of addresses to routes and connections, and the protection of information carried in Internet datagrams. Currently, attackers spend far less energy and time mounting 
attacks than their targets spend defending against them.

Section III introduces a simple approach to ensure that Internet datagrams never loop, even when routing tables contain long-term or short-term routing-table loops. The approach operates by having the FIB entry for an address prefix state the next hop and the hop-count distance to the prefix, and by using the TTL filed of a datagram to enforce an ordering constraint ensuring that a router can forward a datagram only to a next hop that is strictly closer to the intended destination.

Section IV introduces a receiver-initiated address allocation algorithm, a simple address swapping function, and an ondemand routing algorithm operating in the data plane, which together ensure that the origins of Internet datagrams remain anonymous to any routers processing the datagram, and that anonymous sources of datagrams can receive traffic from public destinations over the reverse paths traveled by their anonymous datagrams.

\section{Current Defenses AgAinst DDoS Attacks}

The methods used to launch DDoS attacks today consist of: (a) sending malformed packets to the victims to confuse protocols or applications; (b) disrupting the connectivity of legitimate users by exhausting bandwidth, router processing capacity, or network resources; and (c) disrupting services to legitimate users by exhausting the resources of servers (sockets, CPU, memory, or I/O bandwidth). We address DDoS flooding attacks aimed at disrupting the connectivity and services offered to legitimate users. Four types of defenses against these attacks have been proposed to date [3], [12], [26], [30], [37]: Attack prevention, which aims at stopping attacks before they reach their targets; attack detection, which attempts to identify the existence of attacks when they occur; attack source identification, which tries to locate the source of the attack independently of the information contained in packets used in the attack; and attack reaction, which aims at eliminating or minimizing the impact of attacks.

The attack prevention approaches proposed to date focus on routers filtering IP datagrams with spoofed source IP addresses (e.g., [5], [13], [18], [22], [25], [28]), or routers adding provenance information to IP datagrams The limitations of existing packet-filtering approaches are that: (a) existing filters provide only coarse-grained descriptions of valid source IP addresses; (b) filters are vulnerable to asymmetric routing and the dynamics of routing protocols; (c) it can be difficult to determine which source IP addresses are valid because of the complexity of the network topology; and (d) the exiting approaches used to update filters either incur substantial signaling overhead or are very slow to update filters with new data. Prior approaches based on adding provenance information require too much effort by the attacked network, because they involve the use and dissemination of secret keys to mark IP datagrams, and some even require additional headers.

Several defense approaches have focused on detecting specific DoS and DDoS attacks (e.g., [2], [7], [16], [29], [39]) or anomaly detection (e.g., [10], [21], [40]). These approaches monitor the behavior of specific protocols or the network in an attempt to detect flow anomalies. The key limitation of defense approaches based on detection is that they must rely on a number of assumptions regarding the behavior of legitimate users, and attackers can adopt countermeasures to evade detection. Some DoS attacks can be detected, given that only a few computer systems are attackers and compromised system must behave differently than benign users to exhaust the resources of their targets. However, the problem is far more difficult for DDoS attacks, which involve many compromised hosts that can mimic legitimate users and need not change the normal pattern of protocol traffic to be effective.

Prior approaches for the identification of attack sources have focused on tracing the origins of attacks by explicit signaling or marking datagrams with the paths they traverse (e.g., [9], [31], [33], [34], [35], [41], [42]). Some path marking techniques have also been combined with filtering. The main limitations of these approaches include that: it may be difficult to infer the attack paths in large DDoS attacks; some approaches can consume considerable storage, processing, and communication overhead; some path markings are not entirely unique; and tracebacks and markings become useful only after the attacks have consumed network resources and have reached their targets. Similarly, prior approaches aimed at filtering Internet datagrams with spoofed IP addresses are only partially effective, because they change router behavior to enact filtering without changing the way in which IP source addresses are assigned to Internet datagrams [5], [13], [18], [22], [25], [28], or the fact that datagram forwarding is independent of the distances to address prefixes stated in routing tables.

Approaches that introduce additional information to denote the provenance of a datagram are difficult to implement and cannot be deployed at Internet scale, because they require public key infrastructure (PKI) support. For example, HIP [27] requires a PKI that is globally deployed to prevent attackers from simply minting unlimited numbers of host identifiers used in HIP. AIP [4] on the other hand assumes a flat addressing space that cannot be applied at Internet scale, is vulnerable to malicious hosts creating unlimited numbers of EIDs, and does not offer an efficient way to recovering from compromised private keys corresponding to the AIP addresses of hosts or accountability domains. Similar limitations exist in approaches that add path information (e.g., [8], [23]).

Attack-reaction approaches seek to minimize the damage caused by attacks by protecting bottleneck resources [24]. The mechanisms that have been proposed include reducing the state needed to execute specific communication protocols (TCP in particular [6], [11], [32]); manage resources, shape traffic, and increase the capacity of servers [19], [36]; and secure the communication between confirmed users and servers [20], [38]. Attempting to reduce connection state in TCP is a good objective in all TCP implementation modifications; however, they have not caught on because of the inconsistencies they introduce in the establishment and termination of connections. Approaches that attempt to manage bottleneck resources are not effective, because such resources are shared fairly by DDoS traffic, leaving limited resources for valid users. On the 
other hand, prior approaches that attempt to secure the communication between valid users and resources are overly complex, because they build new mechanisms to enforce provenance and obfuscation on top of existing Internet methods for naming, addressing and routing.

\section{ELIMINATING FORWARDING LOOPS IN THE INTERNET}

The Internet datagram forwarding algorithm is based on FIB entries that simply state the next hops for IP address prefixes. To forward a datagram intended to destination address $d$, an IP router looks up its FIB to obtain the best match for $d$ among the entries listed in its FIB for known IP address prefixes and decrements the TTL of the datagram.

Two approaches are currently used to cope with the occurrence of forwarding loops in Internet forwarding. In the forwarding plane, the time-to-live (TTL) field of an Internet datagram is used to discard a datagram after it circulates a forwarding loop too many times. In the control plane, routing protocols (e.g., OSPF and EIGRP) are used to reduce or eliminate the existence of routing loops. However, even if a loop-free routing protocol is used in the control plane, a datagram may still circulate a forwarding loop while routing tables are inconsistent among routers.

We propose using FIBs to ensure that forwarding decisions in the data plane are consistent with the routing information maintained by the routing protocol operating in the control plane. The FIB entry stored at router $i$ for address prefix $d^{*}$ states the minimum-hop distance $H_{d^{*}}^{i}$ to the prefix in addition to the next hop $n$ to the prefix. We assume for convenience that a distance stated in a FIB is a minimum-hop count to a destination. We denote by $P^{k}\left[s^{k}, d, T^{k}, I D^{o}\right](p)$ an Internet datagram sent by router $k$ with a header that contains a source address of local scope $\left(s^{k}\right)$, a destination address of global scope $(d)$, a TTL value $\left(T^{k}\right)$, and an origin ID $\left(I D^{o}\right)$, plus payload data $p$. Router $i$ uses the following rule to forward such a datagram using its FIB within a network.

\section{TTL-based FIB Rule (TFR):}

Router $i$ accepts to forward $P^{k}\left[s^{k}, d, T^{k}, I D^{o}\right](p)$ from router $k$ towards the best-match prefix $d^{*}$ for $d$ if $T^{k}>H_{d^{*}}^{i}$.

If router $i$ accepts $P^{k}\left[s^{k}, d, T^{k}, I D^{o}\right](p)$, it sets $T^{i}=H_{d^{*}}^{i}$ and forwards datagram $P^{i}\left[s^{i}, d, T^{i}, I D^{o}\right](p)$ to its next hop towards prefix $d^{*}$. A router simply drops a datagram intended for a destination address of global scope with a TTL value that does not satisfy TFR.

TFR consists of imposing an ordering constraint on the traditional Internet datagram forwarding algorithm based on FIB entries, and making the TTL value of the datagram equal to the distance stored in the FIB for the intended destination, rather than simply decrementing its value. The following theorem proves that TFR eliminates IP forwarding loops.

Theorem 1: No Interest can traverse a forwarding loop in an IP network in which TFR is used to forward datagrams.

Proof: Consider a network in which TFR is used and assume for the sake of contradiction that routers in a forwarding loop $L$ of $h$ hops $\left\{v_{1}, v_{2}, \ldots, v_{h}, v_{1}\right\}$ forward a datagram for destination $d$ along $L$ with no router in $L$ detecting that the datagram has traversed loop $L$.

Given that $L$ exists by assumption, router $v_{k} \in L$ must forward $P^{v_{k}}\left[s^{v_{k}}, d, T^{v_{k}}, I D^{o}\right](p)$ to router $v_{k+1} \in L$ for $1 \leq k \leq h-1$, and router $v_{h} \in L$ must forward $P^{v_{h}}\left[s^{v_{h}}, d, T^{v_{h}}, I D^{o}\right](p)$ to router $v_{1} \in L$.

According to TFR, if router $v_{k}(1<k \leq h)$ forwards $P^{v_{k}}\left[s^{v_{k}}, d, T^{v_{k}}, I D^{o}\right](p)$ to router $v_{k+1}$ as a result of receiving $P^{v_{k-1}}\left[s^{v_{k-1}}, d, T^{v_{k-1}}, I D^{o}\right](p)$ from router $v_{k-1}$, then it must be true that $T^{v_{k-1}}>H_{d^{*}}^{v_{k}}$, where $d^{*}$ is the address prefix that is the best match for destination $d$. Similarly, if router $v_{1}$ forwards $P^{v_{1}}\left[s^{v_{1}}, d, T^{v_{1}}, I D^{o}\right](p)$ to router $v_{2}$ as a result of receiving $P^{v_{h}}\left[s^{v_{h}}, d, T^{v_{h}}, I D^{o}\right](p)$ from router $v_{h}$, then it must be true that $T^{v_{h}}>H_{d^{*}}^{v_{1}}$. However, these results constitute a contradiction, because they imply that $H_{d^{*}}^{v_{k}}>H_{d^{*}}^{v_{k}}$ for $1 \leq k \leq h$. Therefore, the theorem is true.

Theorem 1 is independent of whether the network is static or dynamic, or whether routers use single-path routing of multipath routing. The ordering constraint of TFR is essentially the same loop-free condition first introduced in DUAL [14]. The difference between the way in which the ordering constraint is used in TFR and in DUAL is that TFR establishes distancebased ordering in the data plane to forward datagrams based on existing FIB entries, while DUAL establishes distance-based ordering in the control plane to build FIB entries that are then used to determine how to forward datagrams.

As stated, TFR is only applicable within an autonomous system (AS) in which the same routing protocol is used to obtain minimum-hop distances to destinations. However, applying the approach used in TFR across autonomous systems is relatively straightforward. A datagram needs to carry two hop counts, one that states the AS hop count to the destination AS, and another one stating the distance to the intended destination within the same AS or the gateway connecting to the next AS along the path to the destination AS.

\section{ANONYMOUS DATAGRAM FORWARDING}

Since the introduction of datagram packet switching by Baran [1], the identifiers used to denote the destinations and sources of datagrams have had global scope. Today, the FIBs maintained by Internet routers list entries that state the next hop to each known IP address range of global scope.

However, five important observations can be made to argue that datagram forwarding does not have to be limited to Baran's original design. First, the purpose of having addresses in datagram headers is to enable hop-by-hop datagram forwarding based on fast lookups of destination-based routing tables, which does not require addresses to have global, longterm meaning. Second, assigning IP addresses to hosts on a long-term basis as if they were names is not necessary and enables attackers to take advantage of the quasi-static nature of such identifiers. Third, there is no technical reason for the origin of an IP datagram to be the entity that assigns the source IP address of the datagram. Fourth, having the destination of an IP datagram ascertain the provenance of a datagram 
without any assistance from the routing infrastructure imposes too much effort on the destination and is done after datagrams with spoofed addresses have wasted network resources. Fifth, the packet-filtering schemes proposed to date to address DDoS attacks do not take full advantage of the distance information maintained in routing tables.

Our approach consists of using IP addresses of local scope to denote anonymous sources of Internet datagrams, and introducing an on-demand routing algorithm to maintain routes to such addresses. Similar to the approach in [17], we advocate Internet datagram forwarding over symmetric paths in order to support the forwarding of Internet datagrams using IP addresses with local scope. The rest of this section describes the mechanisms for supporting anonymous datagram forwarding in the Internet using a simple example. A formal description of the algorithms is omitted for brevity.

\section{A. Receiver-Initiated Source Address Assignment}

Each router announces a continuous interval of IP addresses (local interval) that the router considers valid local-scope addresses used to denote sources or destinations of Internet datagrams.

Router $i$ maintains a local-interval set table $\left(L I S T^{i}\right)$ that lists the local interval announced by each neighbor router $k$ $\left(L I^{i}(k)\right)$ to router $i$, and the local interval announced by router $i$ to its neighbors $\left(L I^{i}(i)\right)$. All local intervals are of equal length $|L I|$, and hence a local interval is uniquely defined by the IP address at the start of the interval. The start of local interval $L I^{i}(k)$ is denoted by $L I^{i}(k)[s]$. Given this, router $i$ can easily map an IP address of local scope $x \in L I^{i}(i)$ to a corresponding IP address of local scope $y$ that is acceptable to neighbor $k$ with the following bijection, where $\epsilon$ is a constant known only to router $i$ :

$$
y \equiv \epsilon+x-L I^{i}(i)[s]+L I^{i}(k)[s] \quad \bmod |L I|
$$

\section{B. Forwarding Information Stored and Exchanged}

PEAR is transparent to end hosts. Clients and servers simply use IP datagrams without modification. The addresses specified in a datagram sent between a host and router may be of local or global scope. A host that originates datagrams must use an IP address taken from the local-interval set used by the adjacent router.

To forward Internet datagrams from an anonymous datagram source to a destination with an IP address $d$ that has global scope, PEAR uses three addresses to forward a datagram. A source IP address of local scope denotes the originating router of the datagram at each hop of the path towards the destination. An IP address of global scope denotes the intended destination. In addition, an IP address of local scope, which we call the origin ID, is used to denote the host that originated the datagram.

The TTL filed of a datagram is used to enforce TFR at each router to forward a datagram towards the best prefix match $d^{*}$ for destination address $d$ of global scope. A router that receives a datagram from a host sets the TTL field of the datagram to equal its hop-count distance to the destination.

Because the source IP address and the origin ID stated in a datagram have local scope, routers along the path from an anonymous source to a destination with a global-scope address must establish forwarding state pointing back towards the anonymous source in order for datagrams from $d$ to the anonymous source to be forwarded. Additional forwarding tables are needed to accomplish this.

Each router maintains a Hop-Specific Routing Table (HRT) to keep track of the next hops towards destinations denoted by addresses that have hop-specific scope. Router $i$ maintains $H R T^{i}$, which is indexed using hop-specific IP addresses taken from $L I^{i}(i)$. An entry in $H R T^{i}$ states a hop-specific IP address (HIP) used to denote a destination $\left(H I P\left(H R T^{i}\right) \in\right.$ $\left.L I^{i}(i)\right)$, a next hop to that destination, $\left(n\left(H R T^{i}\right) \in N^{i}\right)$, and a hop-specific address mapping used to handle collisions of hop-specific IP addresses $\left(\operatorname{map}\left(H R T^{i}\right) \in L I^{i}(i)\right)$.

$H R T^{i}[H I P, n$, map $]$ denotes a given entry in $H R T^{i}$. A HIP used as the source IP address in a datagram forwarded by router $i$ is denoted by $S H I P^{D}(i)$. Similarly, $D H I P^{D}(i)$ and $O H I P^{D}(i)$ are used to denote the destination IP address and the origin IP of a datagram forwarded by router $i$, respectively.The origin IDs are not stored in HRT entries. Each router uses the same bijection of Eq. (1) to map the origin ID stated in a datagram it receives to the origin ID of the datagram it forwards towards its destination.

Each router also maintains a Destination Routing Table (DRT) to reduce the amount of forwarding state needed in relaying routers. Router $i$ maintains $D R T^{i}$, which indexed using origin IDs received in datagrams and lists entries corresponding to sources of datagrams that have indicated a destination address that is directly attached to router $i$. An entry in $D R T^{i}$ states an origin ID $\left(\left(O\left(D R T^{i}\right)\right)\right.$ and a hopspecific IP address $\left(\left(H I P\left(D R T^{i}\right)\right)\right.$, both of which are in the local interval defined for router $i$.

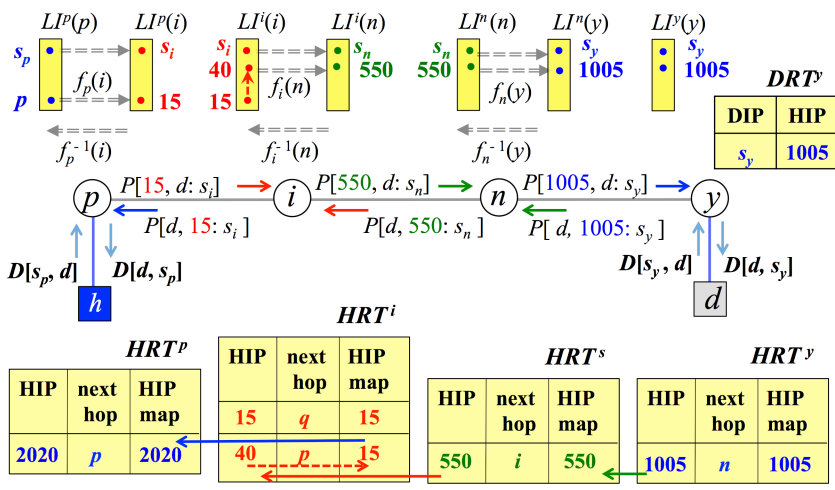

Fig. 1. Datagram forwarding using IP address swapping with PEAR

\section{Forwarding of Datagrams from Anonymous Sources}

Routers that forward a datagram from an anonymous source to a destination address of global scope use their FIBs to 
determine the next hop and establish forwarding state in their HRTs as the datagram is forwarded to allow datagrams to flow back to anonymous sources.

Figure 1 shows an example of the forwarding state established at relay routers between an anonymous source and a well-known destination to enable datagrams to be sent back to IP addresses of local scope. The example in the figure shows a client $h$ initiating communication with a server assigned an address $d$ that has network-wide scope and is part of an address prefix listed in the forwarding information bases (FIB) maintained by routers. It is assumed that the client has obtained the IP address of the intended destination $d$ using the DNS or other means. The IP address assigned to host $h$ is $s_{p}$ and has only local scope at router $p$, i.e., other routers do not associate $s_{p}$ with the same host $h$. In the example, host $h$ must use IP address $s_{p}$ as its source address. Router $p$ accepts datagrams from host $h$ only if the IP source address in those datagrams is $s_{p}$. The TTL field is not shown in the figure, but TFR is assumed to be applied by each relay router.

Starting with the router that receives a datagram from an attached host (router $p$ in the example), routers establish forwarding state using a source IP addresses of local scope, an origin ID that is also an IP address of local scope, and the destination address. In the figure, a datagram forwarded between two routers towards the destination with global-scope address $d$ is denoted by $P\left[x, d: s_{x}\right]$, where $x$ is a source IP address of local scope, $d$ is the global-scope address, and $s_{x}$ is an origin ID. The source IP address and origin ID of a datagram being forwarded by router $r$ to router $n$ along the path from host $h$ to address $d\left(P_{h d}\right)$ are taken from the local interval assigned by $n$ to $r\left(L I^{r}(n)\right)$ using the bijection stated in Eq. (1).

In the example of Figure 1, router $i$ has an exiting entry $H_{R T}{ }^{i}[15, q, 15]$ when it receives datagram $P\left[15, d: s_{i}\right]$ from router $p \neq q$. The destination IP address in the datagram has global scope and hence is forwarded based on its FIB. However, the source IP address in the datagram received from $p$ collides with entry $H R T^{i}[15, q, 15]$. Accordingly, router $i$ selects $H I P=40$, which is not used in any existing HRT entry; creates entry $H R T^{i}[40, p, 15]$, sets $S H I P^{D}(i)=$ $f_{i}(n)[40]=550$ and $O H I P^{D}(i)=f_{i}(n)\left[s_{i}\right]=s_{n}$; and forwards datagram $P\left[550, d: s_{n}\right]$ to router $n$.

When router $y$ receives $P\left[1005, d: s_{y}\right]$ from router $n$, it determines that the host with address $y$ is locally attached. Accordingly, it creates an entry in $D R T^{i}$ with the tuple $\left(s_{y}, 1005\right)$ and passes datagram $D\left[s_{y}, d\right]$ to the host with IP address $d$.

\section{Forwarding of Datagrams to Anonymous Sources}

Routers use their HRTs to forward datagrams to destinations denoted with hop-specific addresses. Given that TFR is used when the entries in HRTs are established, the paths implied by HRT entries are loop-free.

In Figure 1, when router $i$ receives datagram $P\left[d, 550: s_{i}\right]$ from router $n$, the fact that the destination address in the datagram is in its local interval $L I^{i}(i)$ instructs router $i$ to use $H R T^{i}$ for forwarding rather than its $F I B^{i}$. To do so, router $i$ computes the inverse function $f_{i}^{-1}(n)[550]=40$. Using $H I P=40$ as the key in $H R T^{i}$, router $i$ obtains the next hop $p$ and sets $D H I P^{D}(i)=15$. Router $i$ also computes $f_{i}^{-1}(n)\left[s_{n}\right]=s_{i}$, sets $O H I P^{D}(i)=s_{i}$, and forwards forwards $P\left[d, 15: s_{i}\right]$ to router $p$. In turn, router $p$ uses $f_{i}^{-1}(p)$ to obtain the values of the destination address and origin ID for the datagram it should forward. However, given that $p=f_{i}^{-1}(p)[15] \in L^{p}(p)$, router $p$ obtains the local host that should receive the datagram. It computes $f_{i}^{-1}(p)\left[s_{i}\right]=s$ and forwards $D[d, s]$ to the host with IP address $s$.

\section{E. Enforcing Anonymity and Provenance of Datagrams}

It is easy to show that PEAR enforces anonymity of datagrams, in the sense that no intruder can determine the origin of a datagram simply by monitoring traffic over a link and reading the headers of datagrams. The reason an Internet datagram divulges the identity of its source is that source addresses have global scope and hence any relay router or intruder receiving the datagram can determine its origin.

Reducing the scope of a source address to the specific hop where the datagram is being forwarded still allows intruders and relay routers to infer the identity of the source in small networks. However, forcing each relay to use an address from an address space provided by the next hop eliminates the ability of an intruder or the receiving router to infer the true identity of the datagram origin. This is the case even in small networks larger than two routers, unless the topology of the network is such that any traffic sent from a given router must be originated by the router (e.g., a leaf router).

Even tough PEAR uses IP addresses of hop-specific scope to provide anonymity, routers can enforce correct provenance of datagrams by eliminating the ability of hosts or routers to inject arbitrary source IP addresses in datagrams being forwarded among trusted routers implementing PEAR.

An ingress router accepts a datagram from an attached host only if it has a source address deemed valid for that host. Furthermore, the source IP address and origin ID it uses in the datagram it forwards to the next hop must both be in the address space provided by the next-hop router, and the same is true at every hop along the path to the destination. Therefore, it follows that any malicious host or team of routers attempting to inject datagrams with spoofed IP source addresses can be identified by the first trusted router that does not receive datagrams with correct source addresses. Furthermore, malicious datagrams using valid source addresses can be traced back to the first untrusted router injecting the traffic, given the inductive nature of the receiver-initiated source address assignment and the simple bijection used at each hop to swap IP addresses to and from anonymous destinations.

\section{CONCLUSIONS AND Future WORK}

We introduced the first approach for Internet datagram forwarding that eliminates forwarding loops even if routing tables contain routing-table loops. Based on the ability to eliminate forwarding loops, we also presented an approach that 
enables the origins of Internet datagrams to remain anonymous while enforcing the correct provenance of datagrams. As such, the proposed forwarding scheme can be applied to eliminate many of the existing DDoS attacks on the IP Internet, which are enabled by the inability of Internet datagrams to enforce correct provenance.

Additional work is needed to formally describe the specific DDoS countermeasures enabled by PEAR. Equally important, TFR can be applied to inter-domain routing by utilizing two hop counts, one that takes effect across autonomous systems and another one that is used within an autonomous system. However, such cluster-based version of TFR has not been proven to be correct as is the case of TFR [15], [14] and this is an obvious next step.

Lastly, even though we have advocated symmetric paths as additional protection against DDoS attacks [17], the Internet has many asymmetric paths, and the proposed scheme must be extrapolated to the case in which datagrams from $s$ to $d$ follow a different path than datagrams from $d$ to $s$.

\section{ACKNOWLEDGMENTS}

This work was supported in part by the Jack Baskin Chair of Computer Engineering at the University of California at Santa Cruz.

\section{REFERENCES}

[1] P. Baran, "On Distributed Communications: I. Introduction fo Distributed Communication Networks," Memorandum RM-3420-PR, The RAND Corporation, Aug. 1964.

[2] S. Abdelsayed et al., "An Efficient Filter for Denial-of-Service Bandwidth Attacks," Proc. IEEE Globecom '03, 2003.

[3] M. Abliz, "Internet Denial of Service Attacks and Defense Mechanisms," Report No. TR-11-178, Univ. of Pittsburg, 2011.

[4] D. Andersen, "Accountable Internet Protocol (AIP)," Proc. ACM SIGCOMM '08, 2008.

[5] F. Baker, "Requirements for IP Version 4 Routers," RFC 1812, 1995.

[6] D.J. Bernstein, "SYN Cookies," http://cr.yp.to/syncookies.html

[7] R.B. Blazek et al., "A Novel Approach to Detection of Denial-ofService Attacks via Adaptive Sequential and Batch-Sequential ChangePoint Detection Methods," Proc. IEEE Systems, Man, and Cybernetics Information Assurance Workshop '01, 2001.

[8] A. Bremler-Barr and H. Levy, "Spoofing Prevention Method," Proc. IEEE INFOCOM '05, March 2005.

[9] H. Burch and B. Cheswitck, "Tracing Anonymous Packets to Their Approximate Source," Proc. 14th Systems Admin. Conference, 2000.

[10] D.E. Denning, "An Intrusion Detection Model," IEEE Trans. Softw. Eng., 1987.

[11] W. Eddy, “TCP SYN Flooding Attacks and Common Mitigations," RFC 4987, 2007.

[12] T. Ehrenkranz and J. Li, "On The state of IP Spoofing Defense," ACM Trans. Internet Technology, Vol. 9, No. 2, 2009.

[13] P. Ferguson and D. Senie, "Network Ingress Filtering: Defeating Denial of Service Attacks which Employ IP Source Address Address Spoofing," RFC 2827, 2000.

[14] J.J. Garcia-Luna-Aceves, "A Unified Approach to Loop-Free Routing Using Distance Vectors or Link States," Proc. ACM SIGCOMM '89, Aug. 1989.

[15] J.J. Garcia-Luna-Aceves, "A Fault-Tolerant Forwarding Strategy for Interest-based Information Centric Networks," Proc. IFIP Networking '15, May 2015.

[16] T.M. Gil and M. Poletto, "MULTOPS: A Data-Structure for Bandwidth Attack Detection,” Proc. USENIX Security Symposium '01, 2001.
[17] M. Handley and A. Greenhalgh, "Steps Towards a DoS-Resistant Internet Architecture," Proc. ACM SIGCOMM '04 Workshops, Aug. 2004.

[18] C. Jin et al., "Hop-Count Filtering: An Effective Defense against Spoofed DDoS Attacks," Proc. 10th ACM Conf. on Computer and Communications Security, 2003.

[19] F. Kargl et al., "Protecting Web Servers from Distributed Denial of Service Attacks," Proc. 10th Int'l World Wide Web Conference, 2001.

[20] A. Keromytis et al., "SOS: Secure Overlay Services," Proc. ACM SIGCOMM '02, 2002.

[21] R.R. Kompella et al., "On Scalable Attack Detection in The Network," Proc. ACM IMC '04, 2004.

[22] J. Li et al., "SAVE: Source Address Validity Enforcement Protocol," Proc. IEEE INFOCOM '02, 2002.

[23] X. Liu et al., "Passport: Secure and Adoptable Source Authentication," Proc. USENIX '08, 2008.

[24] J.K. Millen, "A Resource Allocation Model for Denial of Service," Proc. IEEE Symposium on Security and privacy, 1992.

[25] J. Mirkovic et al., "Attacking DDoS at The Source," Proc. IEEE ICNP '02, 2002.

[26] J. Mirkovic and P. Reiher, "A Taxonomy of DDoD Attack and DDOs Defense Mechanisms," ACM SIGCOMM CCR, Vol. 34, No.2 , April 2004.

[27] R. Moskowitz et al., "Host Identity Protocol," RFC 5201, April 2008.

[28] K. Park and H. Lee, "On The Effectiveness of Router-Based Packet Filtering for Distributed DoS Attack Prevention in Power-Law Internets," Prof. ACM SIGCOMM '01, 2001.

[29] T. Peng et al., "Proactively Detecting Distributed Denial of Service Attacks Using Source IP Address Monitoring," Proc. IFIP Networkig '04, 2004

[30] T. Peng et al., "Survey of Network-based Defense Mechanisms Countering The DoS and DDoS Problems," ACM Computing Surveys, Vol. 39, No. 1, April 2007.

2011.

[31] S. Savage et al., "Practical Network Support for IP Traceback," Proc. ACM SIGCOMM'O0, 2000.

[32] C.L. Schuba et al., "Analysis of A Denial of Service Attack on TCP," Proc. IEEE Symposium on Security and Privacy '97, 1997.

[33] A. Snoeren et al., "Hash-based IP Traceback," Proc. ACM SIGCOMM '01, 2001.

[34] D.X. Song and A. Perrig, "Advanced and Authenticated Marking Schemes for IP Traceback," Proc. IEEE INFOCOM '01, 2001.

[35] R. Stone, "Centertrack: An IP Overlay Network for Tracking DoS Floods," Proc. 9th USENIX Security Symposium, 1999.

[36] O. Spatscheck and L. Petersen, "defending against Denial of Service Attacks in Scout," Proc. 3th Symposium on Operating Systems Design and Implementation, 1999.

[37] S. Taghavi et al., "A Survey of Defense Mechanisms Against Distributed Denial of Service (DDoS) Flooding Attacks," IEEE Comm. Surveys and Tutorials, Vol. 15, No. 3, 2013

[38] U. Tupakula and V. Varadharajan, "A Practial Method To Counteract Denial of Service Attacks," Proc. ACSC '03, 2003.

[39] H. Wang et al., "Detecting SYN Flooding Attacks," Proc. IEEE INFOCOM'02, 2002.

[40] P.D. Williams et al., "CDIS: Towards a Computer Immune System fore Detecting Network Intrusions," Proc. Int'l Symp. on Recent Advances on Intrusion Detection, 2001.

[41] A. Yaar et al., "Pi: A Path Identification Mechanism to Defend Against DDoS Attack," Proc. IEEE Symposium on Security and Privacy, 2003.

[42] A. Yaar et al., "StackPi: New Packet Marking and Filtering Mechanisms for DDoS and IP Spoofing Defense," IEEE JSAC, 2006. 